\title{
Computer mediated collaborative writing in higher education: enriched communication support using voice annotations
}

\author{
Christine Steeples \\ C SALT \\ Lancaster University \\ United Kingdom
}

\begin{abstract}
New computer based communications technologies make it possible for people to collaborate in their learning, even when remote from one another in space and/or time. The development of computer environments supporting collaborative learning in enriched ways provides the focus for this paper. Multimedia type communication facilities are now available within localized mail systems and word processors. It is possible to integrate voice messages and graphics into documents to send over a local area network. This paper explores the potential value in higher education of voice annotations in a computer mediated collaborative writing task. A small scale study where voice annotations have been used to support collaborative writing processes is described and some initial findings are presented.
\end{abstract}

Main conference themes: flexible learning, distance learning

Educational areas: higher education

Study topics:

Secondary keywords: collaborative learning, communication, multimedia, voice 


\section{INTRODUCTION}

New computer based communications technologies - such as electronic conferencing, electronic mail, communal hypermedia databases and integrated office programmes-make it possible for people to collaborate in their learning, even when remote from one another in space and/or time. The development of computer environments supporting collaborative learning in enriched ways through the use of multimedia provides the goal and context for this research. Whilst research into visual display in the computer interface is extensive, audio research in this area is much more sketchy [1]. This paper is concerned with the use of audio in computer based communication for collaborative writing in educational settings and reports on a recent small scale study of computer mediated collaborative writing

\section{DEVELOPMENTS TOWARDS COMPUTER MEDIATED MULTIMEDIA COMMUNICATION}

There is a number of advantages to be gained in the use of computer mediated communications (CMC) in learning settings. Using CMC participants are not required to be working simultaneously, so everyone has the freedom to work at times which suit them best within agreed periods. This flexibility to break free from the constraints of fixed meetings is persuasive. More subtly CMC can promote opportunities for broader discussion in terms of both available time and the range of participants able to join in. For the individual learner reflection and self pacing are supported [2].

Such communication is however, at present, very largely text based using asynchronous electronic mail and computer conferencing systems. Developments to enable richer communication through computer systems, allowing the integration of not only text, but also images/graphics and audio are only now being realized in usable systems.

Multimedia type communication facilities are available within localized mail systems and word processing software. It is possible to annotate documents with audio or text and include graphics into documents which can be attached to mail messages and sent over a local area network. The foreseeable future should see such integration and enriched communication facilities operating fluidly over wider networks. There is a future for 'learning together, apart' [3] which draws strength from the flexibility computer mediated communication offers (in terms of timing and location) and takes advantage of developments towards broadband communication. 
Research studies need to elicit the value which multimedia communication might offer to remote or distributed learners. The study described in this paper examines the potential of audio information (voice annotations) in multimedia communication amongst collaborating learners engaged in a writing task.

\section{Why collaboration?}

Collaboration can be examined from two perspectives. Collaboration in learning tasks and skills acquired in learning to collaborate.

Collaboration in learning activities can offer a number of benefits to distributed learners. The salient features of collaborative activities advocated by a number of researchers $[4,5,6]$ include that collaboration:

- encourages active and deep engagement in the learning process;

- supports the sharing of individual experience and context;

- requires articulation which can make manifest an individual's understanding and expose deficits;

- promotes the development of metacognitive skills of concept refinement and revision;

- supports the human characteristic of working (and learning) with others.

Learning to collaborate is part and parcel of the process of learning to work with others demanding the integration of subject specific skills with more generally applicable communication and coordination skills. These are skills required for effective cooperation and communication in work and increasingly in learning settings.

Sharing and discussion with colearners around collaborative tasks aids the articulation of individual viewpoints. Computer supported collaborative learning facilitates this. In this context a first small scale investigation with groups of students across the university has provided some general understanding about the usefulness of voice annotations.

\section{Using voice annotations for enriched communication support}

Sound and vision are complementary forms of communication. Audio may be used to enrich or to ease the use of visual information in learning settings. Voice annotated representation may allow the user to focus attention on the visual representation while listening to the commentary of another user. This gives a different perspective on the information while retaining visual attention. Voice annotations convey information which is difficult or awkward to display in text, graphics or is difficult to visualize. These may also reduce visual clutter in the computer interface.

The audio channel can also present semantically identical information. This redundancy effect can promote the processing of information and so aid 
learning [7]. Additionally the use of voice can allow a more expressive, personal style of communication.

\section{Voice annotations in creative writing processes}

Much of the learning activity in Higher Education revolves around, or is made manifest in, written documents. Writing can be an extremely difficult task requiring tremendous mental effort. A valuable contribution of voice annotations could be easing of the cognitive load in short term or working memory in the process of writing. The writer could use the voice annotation facility to record his ideas, while struggling to find the exact wording or working through conflicting ideas.

Collaborative writing tasks involve considerable social and intellectual complexity. Authors need to find mutually acceptable ways in working towards a coherent text. They need to negotiate and assume responsibilities. They need to coordinate their activities and develop a shared understanding. Collaborative writing processes have been studied extensively and more recently the technologies employed to support these processes, e.g. $[8,9,10]$.

In deciding how to organize and coordinate a collaborative writing task three main patterns of practice have been identified. Of course there can be subtle variations, but typically each person chooses to take responsibility for a section of the task, efforts are exchanged and blended into the final document. Alternatively, the document is written entirely together. A further pattern can be the editor-in-chief coordinating the contributions of others and responsible for final revision.

In any of these practices voice annotations can be useful in aiding the creative process and in supporting coordination of activities. The annotations might provide a more personal way to communicate and to receive complex, equivocal or controversial information. Kraut et al [11] suggest that using voice not only allows the speaker to express his thoughts in a personal way, but also does cause the speaker to consider the audience-the person(s) who will listen to their message. This is clearly important to fostering of productive, collaborative working relationships.

Voice annotations can provide an easier and quicker way to express, explain or justify a revision made. It is often easier to say rather than write and for many it is quicker to speak than to type in text.

Voice annotations can be used to give contextual or background information to share with collaborators, but not necessarily to include in the actual text. By voice annotation the flow of reading through the text is not disturbed.

Writers have commented upon the need to maintain a global view of the text they are trying to achieve. Working with a word processor can make this global view, the visual 'picture' of a text, difficult. Here voice annotations can 
supply high level or metacomments replicating outliners or advance organizers about the goals, purposes and structuring of the document. These would not impede visual scanning of the text or require visual switching to another text.

Uninterrupted flow in reading can be important when creating a group based document. A reader will want to attend to a new section of text before observing the comments of others. Multiple insertions in the text make a document very messy to read. Audio icons can here give the visual signal at the point of issue (or close to it) that there is an added comment. The reader can make his own choice whether to access the annotation or to ignore it.

Suggested use of voice annotations in creative processes might lead to the assumption that the annotation has a limited value-once the document is completed the annotation(s) are redundant. But reusing the document including the annotations can provide a rich learning resource for helping other learners in their writing practices. The annotations can give cues to how the document evolved, how ideas developed, how conflicts were resolved and give background or contextual explanation.

\section{STUDY 1: VOICE ANNOTATIONS TO SUPPORT COMPUTER MEDIATED COLLABORATIVE WRITING PROCESSES}

The first of a set of investigations has recently been completed. This study has examined the uses made of voice annotations to support collaborative document production processes.

\section{Description of the study}

The goal of this study was to look at the value of voice annotations in terms of process support, ease of communication and resolution of conflicts. The study gathered data on how individual users chose to use audio and textual annotations. The study compared audio or textual annotations in their ease of use, their speed of creation, their effect on reading through drafts and comments or revisions. It examined the effectiveness of audio in supporting and resolving equivocal communication. The study looked closely for examples of audio used to quickly capture ideas and ease cognitive load in working memory. It explored the users' experiences and value they might suggest for annotations in collaborative learning processes.

\section{Subjects}

The study involved groups of three students studying on the same course in a collaborative writing task. The task required the group to author a review of a 
reading used on their course. The task is generic and groups from several university departments were recruited.

The review required a descriptive synopsis of the reading, a summary of the key issues and ideas raised together with their own reflection upon those themes and ideas. The groups were asked to write their review as an aid to future students studying the same reading.

Eight groups (of three people) were allocated to three conditions. Using three groups to each condition gives just a small sample and there is no expectation of an even distribution of practices. Rather, the study has looked for interesting practices emerging in the use of voice annotations and the variables associated with those practices. The three conditions applied were:

- Using textual annotations only (Condition T);

- Using textual and voice annotations with suggested uses for annotations given (Condition T/A);

- Using audio and textual annotations with entirely free choice (Condition $\mathrm{T} / \mathrm{A}+)$.

In the second condition above (Condition T/A) the groups were given some suggestions of appropriate use of the text and voice annotations derived from the literature. It was suggested that text annotations are useful for simple and localized comments such as sentence structure or grammatical errors. It was suggested voice annotations would be useful for complex comments relating to global issues in the writing. It was also suggested that voice annotations would be useful for helping resolve conflict or differences in opinion amongst the group by virtue of their expressive nature.

\section{Technical}

The groups created their review using word processing software on a Macintosh computer connected to a local mail server. After the initial face-toface meeting of the group all communication was computer mediated using Word 5.1 $1^{\mathrm{TM}}$ and Microsoft Mail ${ }^{\mathrm{TM}}$. Word 5.1 provides the facilities to insert text and voice annotations. Microsoft Mail and Word are integrated so that documents can be sent as attachments to mail messages.

An introductory technical training session was given. The text annotation facility (see Fig. 1 below) was introduced to all participants. Training in using the microphone and recording voice annotations (see Fig. 2 below) was given to students in conditions T/A and T/A+. Guides to the software and recording equipment were provided to each student. Technical support was constantly available during the study. 


\section{Data}

Each participant completed a questionnaire to gather background data on their study details, age, computer experience and group working skills. Each group then began the task with a face-to-face meeting to negotiate and coordinate their specific responsibilities. Each initial meeting was recorded on videotape.

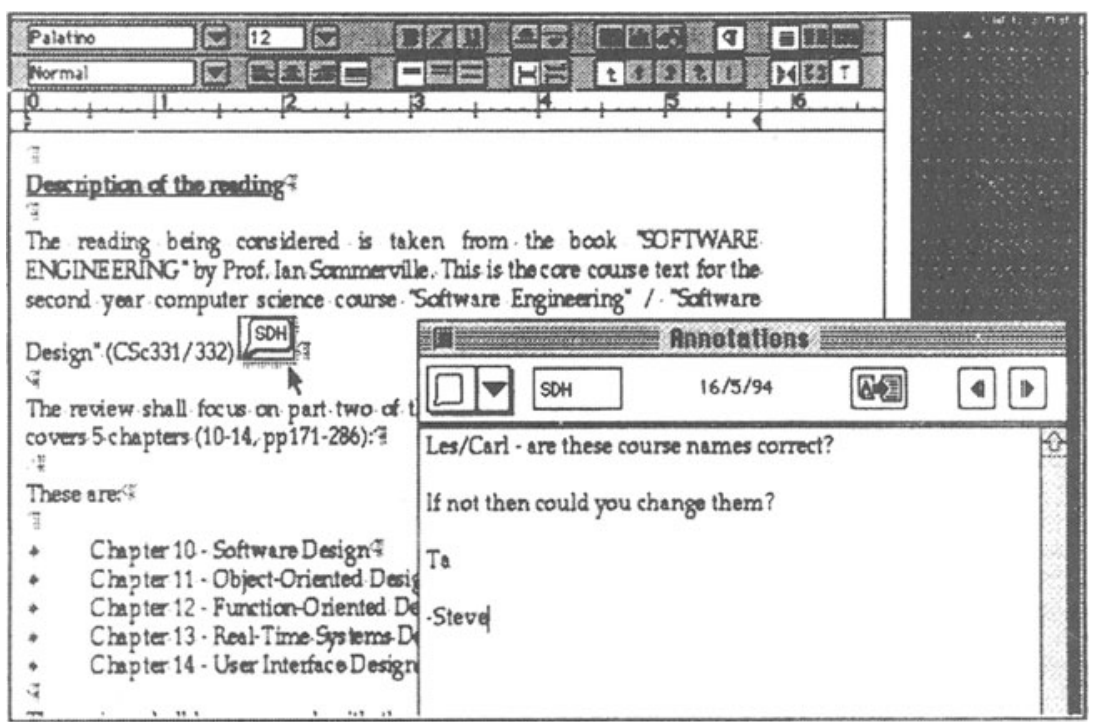

Fig. 1 Text annotation (pointer indicates the annotation icon in the document)

Each group was assigned 9 hours over a 3 week period to use the computer and complete the review. It was entirely up to each group how they used or divided out the computer time, but they had to use it singly and for all their communication. In each computer session the students were asked to 'think aloud', to talk through what they were doing and why. These sessions were videotaped. As the drafts were electronically circulated among the group a copy (with its annotations) was automatically routed to a central store as part of the data capture. A copy of the completed review was also collected.

A post study interview gathered experiences and comments from the participants. It gave the opportunity to clarify any issues raised by the questionnaires or immediately apparent from the videotaped working sessions and any significant comments could be explored. 


\section{Initial findings from Study 1}

Reaction to using voice annotations has been mixed. There were some who could only see the voice annotations as a novelty and there were at least two participants who were disinclined or embarrassed about recording their voices.

Aural scanning was problematic to some of the participants. The serial nature of audio means that time is needed to replay and listen to a message whereas a textual message can be visibly scanned much quicker under user control and in a more customary manner. Some of the listeners found that they needed to relisten to the voice annotations. This was mainly attributed to the design of the interface and the icon used as the visual referent to the voice annotation. A speaker symbol in the document text is the only clue to the voice annotation (see Fig. 2). The symbol gives no immediate information about the content or creator of the voice annotation. The listener receives no indication that this might be an annotation that they have already listened to. Indeed the limitations of voice annotations seem to be primarily related to listening to audio, as others have also observed e.g. [12].

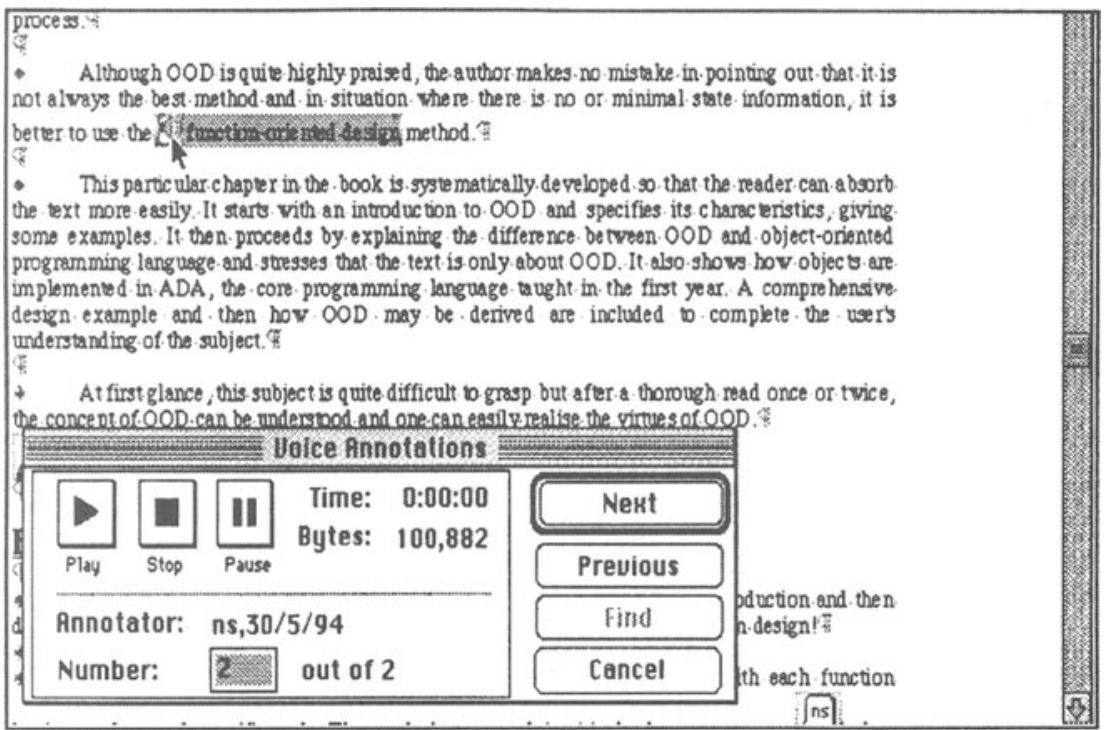

Fig. 2 Voice annotation control panel (pointer indicates the speaker symbol icon in the document) 
There were other instances where participants showed enthusiasm for voice annotations, found them a more sociable medium for communication and discovered valid purposes for voice in collaborative writing processes.

One subject said she had valued the voice annotations "to help her find the right words" with the aid of the others in her group. She expressed that she found it easier to say what she meant rather than to write out her thoughts and ideas. Another agreed about the benefits of rapidly capturing ideas for 'spontaneous' communication, adding: "if it was there in my head I could just say it ... to get out what I had in my head at the time".

Another student described how using a voice annotation had made it easy for him to orally explain to his colleague how he had created a diagram in the text. He could explain while visually looking at the screen, as his colleague could listen to the explanation while studying the screen display. Being able to scan a written text while listening to an auditory comment, was suggested as useful by another subject.

The communicative nature of voice annotations was strongly upheld by the subjects in this study. All voice annotations were used to express ideas and give comments to others rather than to help individuals sort out their own thoughts. The subjects suggested the voice annotations had supported their working as a group, particularly in coordinating the writing task. The voice annotations were most used at the stages of developing and revising first drafts.

\section{SUMMARY}

This paper has introduced research about audio (voice) annotations used to support computer mediated collaborative learning processes and has endeavoured to begin discussions about the unique contribution audio can make in collaborative contexts.

Detailed analysis of the study reported here is still under way, but participants' comments do support the suggestions put earlier in this paper. Namely, voice annotations can be useful for articulating complex ideas, for retaining attention to visual information, to explain visual information and to support (and retain the social nature of) group communication.

Current practices in working with computers have been built around a monomedial, text based, visual display and we are only beginning to replicate more naturalistic communication with the integration of visual, nontextual representations and the use of audio including voice. These changes require the development of new ways of working with computers: a relearning of what we would naturally do when working and learning with others. 


\section{REFERENCES}

1. Kramer, G. (1994) An introduction to auditory display. In Auditory display (ed Kramer, G.), Addison-Wesley, Reading, MA.

2. Steeples, C., Goodyear, P. and Mellar, H. (1994) Flexible learning in higher education: the use of computer-mediated communications. Computers and Education 22 (1/2) pp. 83-90.

3. Kaye, A. R. (1992) Learning together apart, in Collaborative learning through computer conferencing: the Najaden papers. (ed Kaye, A. R.), Springer-Verlag, Berlin, pp. 1-24.

4. Harasim, L. (1990) Online education: an environment for collaboration and amplification, in Online education: perspectives on a new environment, (ed Harasim, L.), Praeger, New York

5. Brown, A.L. and Palinscar, A.S. (1989) Guided cooperative learning and individual knowledge acquisition, in Knowing, learning and instruction: essays in honor of Robert Glaser, (ed Resnick, L.B.), Lawrence Erlbaum, Hillsdale, NJ, pp. 393-452

6. Scardamalia, M. and Bereiter, C. (1992) An architecture for collaborative knowledge building, in Computer-based learning environments and problem solving (eds de Corte, E. et al), Springer-Verlag, Berlin, pp. 41-66.

7. Aarntzen, B.M.H. (1994) Audio in interactive tutorial courseware: methodological and design guidelines. Doctoral dissertation, Enschede, The Netherlands.

8. Chalfonte, B. Fish, R and Kraut, R. (1991) Expressive richness: a comparison of speech and text as media for revision. ACM/SIGCHI'91 Human Factors in Computer Systems, pp. 21-26.

9. Kellogg, (1985) Computer aids that writers need. Behaviour Research Methods, Instruments \& Computers 17 (2) pp. 253-258.

10. Rimmershaw, R. (1992) Collaborative writing practices and writing support technologies. Instructional Science 21, pp. 15-28. 

communication support using voice annotations

11. Kraut, R., Galegher, J., Fish, R. and Chalfonte, B. (1992) Task requirements and media choice in collaborative writing. Human-Computer Interaction 7, pp. 375-407.

12. van Nes, F. (1992) Design and evaluation of applications with speech interfaces - experimental results and practical guidelines, in Methods and tools in user-centred design for information technology (eds Galer, M., Harker, S. and Ziegler, J.) Elsevier Science, North Holland, pp. 281-297. 